\title{
Diabetes and musculoskeletal disorders-a review
}

\begin{abstract}
Diabetes mellitus comprises of metabolic diseases that are characterized by elevated levels of blood glucose or hyperglycaemia, which is a result of insulin deficiency, insulin resistance or both. Marked hyperglycaemia causes an increase in morbidity and mortality that are related to macrovascular and microvascular complications. Musculoskeletal disorders seem to occur often in patients who have diabetes. Musculoskeletal pain is a usual occurrence in patients who have diabetes compared to the general population. The aim of this review was to explore and musculoskeletal disorders in diabetes. Diabetes causes significant morbidity and mortality and has been found to have an association with musculoskeletal disorders. A number of studies have found that diabetes has an association with musculoskeletal disorders such as osteoarthritis, rheumatoid arthritis, osteoporosis, fibromyalgia and carpal tunnel syndrome however the reasons underlying them are unclear. This review looks at the effects of diabetes on musculoskeletal disorders and the mechanisms through which diabetes contributes to them, so as to have a better understanding of the impact they may have in patients with diabetes. Further studies are necessary to understand how diabetes correlates with connective tissue metabolism, cytokines and obesity, for better patient care, treatment and prevention.
\end{abstract}

Keywords: diabetes mellitus, hyperglycaemia, osteoarthritis, fibromyalgia, carpal tunnel syndrome, musculoskeletal disorder
Volume 7 Issue 2 - 2020

\author{
Shraboni Ghosal,' Arnab Ghosal' \\ 'Nutrition Consultant, UK \\ ${ }^{2}$ B.Sc. Biology, Staffordshire University, UK
}

Correspondence: Shraboni Ghosal, Nutrition Consultant, Newcastle under Lyme, Staffordshire, UK, Email castlediet।@gmail.com

Received: June 10, 2020 | Published: June 29, 2020
Abbreviations: DM, diabetes mellitus; WHO, World health organization; T1DM, type 1 diabetes mellitus; T2DM, type 2 diabetes mellitus; PWD, patients with diabetes; IR, insulin resistance; OA, osteoarthritis; RA, rheumatoid arthritis; $\mathrm{CF}$, charcot foot; $\mathrm{AC}$, adhesive capsulitis, LJM, limited joint mobility; FMS, fibromyalgia syndrome; DC, Dupuytren contracture; TF, trigger finger; CTS, carpal tunnel syndrome; ORs, odds ratios; COA, charcot osteoarthropathy; HR, hazard ratio; FMS, fibromyalgia syndrome; DISH, diffuse idiopathic skeletal hyperostosis; FS, frozen shoulder; RR, relative risk; AGEs, advanced glycation end products; RAGE, receptor for advanced glycation end products; TNF, tumor necrosis factor; IL, interleukin; QoL, quality of life

\section{Introduction}

Diabetes mellitus (DM) comprises of metabolic disorders that are chronic and characterized by hyperglycaemia, which is a result of insulin deficiency, insulin resistance or both. ${ }^{1}$ Marked hyperglycaemia causes increased morbidity and mortality, which are mostly associated with macrovascular and microvascular complications. ${ }^{2}$ The World Health Organization (WHO) estimated that globally about 422 million people currently have diabetes. ${ }^{3}$ The number of adults with diabetes globally has been estimated to increase to 592 million by $2035 .{ }^{4}$ In the United States (US), diabetes affects about $10 \%$ of the general population. ${ }^{5}$ In 20092.6 million people were diagnosed with diabetes in the UK. ${ }^{6}$ Diabetes can be grouped into two main categories. The autoimmune destruction of beta-cells of the pancreas causes Type 1 diabetes mellitus (T1DM), which starts in the young. ${ }^{7}$ The most common form is Type 2 diabetes mellitus (T2DM) that is characterized by insulin resistance (IR) and comprises about $90-95 \%$ of patients with diabetes (PWD). ${ }^{6}$ Evidence suggests that a number of musculoskeletal disorders are linked with diabetes, which may cause significant pain and disability. ${ }^{8-10}$ While musculoskeletal disorders in PWD have been reported in previous studies, ${ }^{11-13}$ the causes underlying them are unclear. This review discusses some of the musculoskeletal disorders associated with diabetes.

\section{Diabetes and musculoskeletal disorders}

Musculoskeletal disorders are common and the prevalence is raised in PWD. ${ }^{14}$ About $20-33 \%$ of people globally and about one in two adults were found to have a musculoskeletal disorder in the US. ${ }^{15}$ Worldwide, in 2017, musculoskeletal disorders were about $16 \%$ of global disabilities. ${ }^{16}$ The common musculoskeletal disorders include osteoarthritis (OA), rheumatoid arthritis (RA), Charcot's foot (CF), adhesive capsulitis (AC), osteoporosis, limited joint mobility (LJM), fibromyalgia syndrome (FMS), Dupuytren's contracture (DC), trigger finger (TF) and carpal tunnel syndrome (CTS). ${ }^{17}$ Musculoskeletal pain and loss of function can be caused due to restricted movement, flexibility and abilities. ${ }^{18}$ In PWD, musculoskeletal pain may have negative consequences, which includes inadequate glycaemic control and a reduction in being physically active. ${ }^{19}$

\section{Diabetes and musculoskeletal pain}

In PWD, several musculoskeletal disorders have been related, that have an impact on all age groups. There is evidence that musculoskeletal pain is a frequent problem in PWD. ${ }^{20}$ In the UK, about 9.3 million working days are lost as a result of pain in the back, hips or knees. ${ }^{21}$ Diabetes has been found to have significant associations with back/lower back pain, pain in the limbs, OA, osteoporosis, RA and shoulder/neck pain, with odds ratios (ORs) ranging between 1.2 to $1.6(\mathrm{p}<0.01) .{ }^{22}$ In a previous study on 950 patients with $\mathrm{T} 2 \mathrm{DM}$, musculoskeletal pain was 1.7 to 2.1 times as frequent $(p<0.001)$ to age and gender matched controls and the pain was more frequent in females $(\mathrm{p}<0.001) .{ }^{20}$ Another study found that there was a significant rise in the pain intensity $(\mathrm{p}<0.001)$ of PWD with knee OA and raised synovitis scores were found $(\mathrm{p}=0.024)$ compared to patients without diabetes. $^{23}$ 


\section{Diabetic neuropathy}

Diabetic neuropathy has been found to affect over $90 \%$ of $\mathrm{PWD}^{24}$ and is a common complication of both T1DM and T2DM. It has been observed in those who have had the disease for 10 years or longer. One of the main symptoms of diabetic neuropathy is pain. ${ }^{22}$ Peripheral neuropathy, retinopathy, autonomic neuropathy or renal failure may be due to the long-term complications of diabetes. ${ }^{2}$ Limited joint mobility (LJM) has been found to be more prevalent in patients with diabetic neuropathy. The blood vessels may be affected by autonomic neuropathy, which leads to increased blood flow and cause inflammation and damage to the bones. Autonomic neuropathy has been found in patients with Adhesive capsulitis (AC) often compared to other PWD, which could be an underlying mechanism. ${ }^{25}$ Sharp pains, numbness, cramps, burning, tingling or sensory loss have been observed in peripheral neuropathy, ${ }^{26}$ which may increase the risk of some forms of OA in PWD. ${ }^{1}$

\section{Charcot osteoarthropathy}

Charcot osteoarthropathy (COA) or neuropathic arthropathy is also known as Charcot's Joint, and is a result of diabetic peripheral neuropathy. ${ }^{27}$ In PWD, it causes damage and deformities of joints along with symptoms such as tingling, numbness or sensory loss. ${ }^{27}$ $\mathrm{COA}$ is a progressive and degenerative disease and pain/discomfort may be observed in the acute stages. Joints such as the knees, ankles and feet, which are the weight-bearing joints, are mostly affected ${ }^{28}$ and people of both genders are equally affected. The incidence of arthropathy was found to be about $10 \%$ in the foot and ankle, with an increase in prevalence of $1 / 680$ in PWD, which increased with the duration of diabetes. ${ }^{18,29} \mathrm{COA}$ is mostly found in people above 50 years $^{18}$ and has been found in $0.1-5 \%$ of PWD. It is caused due to a lowering of the afferent neural impulses, which leads to abnormal vasomotor regulation. ${ }^{30}$ T2DM was found in about two-thirds of people with $\mathrm{COA} .^{30,31}$

\section{Osteoarthritis}

Osteoarthritis (OA) is caused due to degradation of the cartilage in one or more joints, which make them stiff and painful. ${ }^{1,32} \mathrm{OA}$ affects around 8 million people in the $\mathrm{UK}^{32}$ and is the most common form of arthritis. ${ }^{22,33} \mathrm{OA}$ commonly affects the knees, hips and joints in the hands and musculoskeletal pain may arise in PWD. ${ }^{1}$ The prevalence of OA amongst PWD was $29.5 \pm 1.2 \%$ and diabetes amongst patients with OA was $14.4 \pm 0.1 \% .{ }^{34}$ In PWD, the overall risk of OA as reported in a systematic review was 1.46 (95\% confidence interval (CI) 1.08, 1.96; $\mathrm{p}=0.01$ ) and diabetes in patients with OA was 1.41 (CI 1.21, 1.65; $\mathrm{p}<0.001) .{ }^{34} \mathrm{~T} 2 \mathrm{DM}$ has been significantly associated with OA (OR $1 \cdot 21,95 \%$ CI $1 \cdot 02,1 \cdot 41) .{ }^{35}$ A cohort study showed that in men with T2DM and knee OA, joint space narrowing was significantly higher than controls. ${ }^{36}$ These results were supported by a few animal studies, which also found a negative effect of diabetes on OA. ${ }^{37}$ In contrast, no association was found between OA and T2DM in those who had knee or hip joint replacement (OR 1.0, 95\% CI $0.5,1.9) .{ }^{38}$ The researchers concluded that diabetes could be a risk factor for hip and knee OA. ${ }^{38}$ Studies have suggested that T2DM may have a pathogenic effect on OA through major pathways involving inflammation and oxidative stress, caused by persistent hyperglycemia and IR. ${ }^{39}$

\section{Rheumatoid arthritis}

Rheumatoid arthritis (RA) is a chronic, inflammatory and autoimmune disease. ${ }^{32,40}$ It affects the synovial membranes of joints and may lead to joint deformities. ${ }^{41}$ Peripheral and symmetric polyarthritis observed in $\mathrm{RA}^{41}$ are likely to cause destruction of cartilage by the immune system. ${ }^{42}$ Inflammation, pain, and decreased function of the affected joints are the common symptoms of RA. ${ }^{43}$ RA has an estimated global prevalence of $0.24 \%$ and females are twice as likely to have RA as males. ${ }^{44}$ Approximately 400,000 people are affected by RA in the UK. ${ }^{32,42}$ An increased prevalence of diabetes has been observed in patients with RA. ${ }^{45,46}$ Diabetes was associated with $55 \%$ elevated odds of having RA in a Danish study. ${ }^{22}$ In contrast, some studies have found no evidence that RA occurs often in PWD. ${ }^{47,48}$ Some studies have shown that T2DM increases the risk for developing RA and conversely, having RA increases the risk for developing T2DM. Patients with RA may be at an increased risk for IR and T2DM. ${ }^{49}$ However, others have suggested that people with T1DM are likely to have a higher chance of developing RA, ${ }^{42}$ as they share some common genetic characteristics such as the chromosomes HLA-DR9, 4q27, PTPN22 and the regions IDDM8 and IDDM5. ${ }^{50}$

\section{Gout}

Gout is an inflammatory arthritis and commonly affects adult males. ${ }^{18}$ The collection of monosodium urate crystals leads to inflamed and painful joints along with acute inflammatory attacks. ${ }^{32}$ Gout had an association with an increased risk of diabetes (hazard ratio [HR] $1.45,95 \% \mathrm{CI} 1.37,1.54)$ and the risk of incident diabetes was more in females (HR 1.77, 95\% CI 1.51, 2.09) than males (HR $1.41,95 \%$ CI $1.33,1.50) .{ }^{51}$ The incidence rates of diabetes were 9.5 in males and 10.1 in females per 1000 person-years in people with gout in a population-based study and 7.2 in males and 5.6 in females per 1000 person-years in controls..$^{52} \mathrm{~A}$ recent prospective study in the UK found that diabetes was related to a lower risk of incident gout ${ }^{53}$ whereas a Chinese study found no significant relationship. ${ }^{54}$ Some studies have found that people with gout are significantly more likely to develop T2DM than controls. ${ }^{55}$ The prevalence of gout in T2DM patients was $22 \% .{ }^{56}$ In gout, serum uric acid levels have been related with the risk of developing diabetes. ${ }^{57}$ Diabetes was strongly related with serum urate concentrations, abdominal obesity and gout, ${ }^{18}$ while others found gout related with diabetes when hyperlipidaemia and obesity were present. ${ }^{47}$

\section{Diabetic muscle infarction}

Diabetic Muscle Infarction is an uncommon complication of longstanding diabetes. ${ }^{58,59}$ with established vasculopathy. The symptoms include sudden pain and inflammation in the lower limbs and it mostly affects the adductor muscles, quadriceps femoris and calf muscles. ${ }^{18}$ Out of $114 \mathrm{PWD}$, it was found to be more common in females (61.53\%), 59.1\% had T1DM and 23.8\% had T2DM..$^{59}$ The underlying factors such as vascular endothelial damage and diffuse diabetic microangiopathy may cause diabetic muscle infarction. ${ }^{60}$

\section{Diabetic amyotrophy}

Diabetic Amyotrophy (Diabetic Cachexia) is a disabling and different form of diabetic neuropathy, ${ }^{61}$ with an estimated incidence of about $1 \%$ amongst PWD. ${ }^{62}$ The symptoms commonly include loss of tendon reflexes, pain, muscle wasting and weakness. ${ }^{18}$ Diabetic amyotrophy has been found to often occur in older males with T2DM and is linked with weight loss. ${ }^{62}$ The underlying factors may be due to a combination of metabolic imbalances, autoimmunity, microvascular inadequacy and oxidative stress. ${ }^{18}$

\section{Fibromyalgia syndrome}

Fibromyalgia or Fibromyalgia syndrome (FMS) is a chronic condition that affects about $2-4 \%$ of adult females. ${ }^{63}$ The symptoms consist of extensive musculoskeletal pain, stiffness and tenderness 
along with functional and cognitive impairments, headaches and tiredness. ${ }^{64}$ Diabetes may have a role in the development of FMS as it was found to be more common in FMS patients than controls. ${ }^{64}$ Peripheral neuropathy has been linked with FMS as it was found in $61.9 \%$ of T2DM patients with FMS compared to only $2.5 \%$ of T2DM patients without FMS. ${ }^{64}$ Other than endocrine and neurological factors, research indicates that environmental and genetic factors may also play a role in the development of FMS. ${ }^{64}$

\section{Diffuse idiopathic skeletal hyperostosis}

Diffuse idiopathic skeletal hyperostosis (DISH), Forestier disease or ankylosing hyperostosis mostly affects the neck or spine. ${ }^{65}$ In $\mathrm{DISH}$, the tendons and ligaments harden along with the formation of new bones. Stiffness or a decrease in movement has been observed in PWD. ${ }^{65}$ DISH commonly occurs in PWD (13-49\%) compared to controls (1.6-13\%) ${ }^{18}$ and another study reported that it was present in $25 \%$ amongst 428 PWD. $^{7}$ DISH is mostly found in T2DM compared to T1DM and mainly occurs in the elderly. ${ }^{7,66}$ DISH affects more males than females and in those with dyslipidaemia, hyperuricaemia or obesity. ${ }^{66}$

\section{Dupuytren's contracture}

Dupuytren's contracture (DC) is a disorder that causes hardening, thickening, tethering and contraction of the fingers, with limited joint movements. ${ }^{67}$ It is associated with peripheral neuropathy and diabetes was found to be a significant risk factor (mainly T1DM) for DC. ${ }^{68}$ DC occurs more in PWD than in the general population ${ }^{69,70}$ and has been reported in $16-42 \%$ of PWD. ${ }^{7,70}$ A systematic review found an association between DC and diabetes (OR 3.06, 95\% CI, 2.69, 3.48). ${ }^{71}$ $\mathrm{DC}$ is common in patients who have long- standing diabetes and may increase with age and the duration of diabetes. ${ }^{72} \mathrm{~A}$ study reported that the prevalence increased in both T1DM and T2DM compared to controls ( 35.0 and $30.0 \%$ versus 6.7 and $10.0 \%$ ), both $\mathrm{p}<0.01$ and had a correlation with age $(\mathrm{p}<0.05){ }^{73}$

\section{Adhesive capsulitis}

Frozen shoulder (FS) or adhesive capsulitis (AC), is a disabling and common musculoskeletal disorder. The symptoms include restricted and painful shoulder movements, mainly with abduction and external rotation that causes acute pain, tightening and stiffness in PWD. ${ }^{22}$ The prevalence of AC was estimated to be $11-30 \%$ in PWD and $2-10 \%$ in controls. ${ }^{74}$ Another study reported that the estimated prevalence was $10-20 \%$ in T1DM, $7-32 \%$ in T2DM and $0-10 \%$ in controls. ${ }^{75}$ In AC, a lower BMD was found in T1DM, whereas patients with T2DM and hyper insulinaemia were found to have a normal/high BMD.$^{65}$ In T1DM, AC was found to be related with age and duration of diabetes whereas in T2DM, it was related only with age. ${ }^{75}$

\section{Osteoporosis}

Osteoporosis is a disorder that causes bone damage and deterioration, making them prone to fracture. ${ }^{26}$ Osteoporosis affects around 3 million people in the UK. ${ }^{76}$ Diabetes has been associated with an increased risk of fracture. Studies suggest that and diabetes could be a risk factor for fracture. ${ }^{26}$ Osteoporosis can cause musculoskeletal pain and bone loss is greater in patients with inadequately controlled diabetes than those with good control. ${ }^{77}$ T1DM and T2DM have different effects on BMD but share common pathways, which cause bones to become fragile. ${ }^{26}$ In the Nurses' Health Study, 1,398 females had a hip fracture during 2.22 million person-years of follow-up and in T1DM, the risk of hip fracture was found to be six-fold higher than those without diabetes. ${ }^{77}$ The age-adjusted relative risk (RR) of hip fracture in T1DM was 7.1 (95\% CI 4.4, 11.4) and 1.7 (CI 1.4, 2.0) in T2DM compared with females without diabetes. ${ }^{77}$ Researchers have suggested that people with T1DM may have an increased risk of osteoporosis because they tend to have a lower BMD. ${ }^{76}$

\section{Diabetic cheiroarthropathy}

Diabetic cheiroarthopathy, Diabetic Hand Syndrome or Limited joint mobility (LJM) is a disabling condition that may occur due to vascular insufficiency ${ }^{64}$ In LJM, limitations occur in joint movements due to contractures and skin of the hands become thick, tight and waxy, with deformities and pain in the joints. ${ }^{78}$ The prevalence of LJM was found to vary from about 8 to $58 \%$ in T1DM, 25 to $76 \%$ in T2DM and 1 to $20 \%$ in controls. ${ }^{65} \mathrm{LJM}$ is associated with age and duration of diabetes. ${ }^{71}$ Researchers have suggested that the differences in the prevalence estimates in LJM may be due to differences in glycaemic control. ${ }^{79}$ The prevalence of LJM in T1DM was found to be related to age $(p<0.05)$ and with duration of diabetes ${ }^{73}$ and some studies have suggested that genetic factors could have a role in the development of LJM. ${ }^{65}$ A study showed that the prevalence of LJM was $8 \%$ in T1DM, $9 \%$ in their siblings without diabetes and $2 \%$ in controls who were not siblings. ${ }^{80}$ In contrast, another study found that genetic factors did not have a role in LJM in patients with T1DM and their first-degree relatives. ${ }^{81}$

\section{Carpal tunnel syndrome}

Carpal tunnel syndrome (CTS) is a neuropathic disorder, which may occur due to diabetic neuropathy and/or compression of the median nerve in the carpal tunnel. ${ }^{7}$ CTS causes pain, tingling and numbness in the hands. The prevalence of CTS in adults ranges from about $2.7 \%$ to $5.8 \%{ }^{82}$ while the incidence is about 125 per 100,000 person-years. ${ }^{61}$ CTS is more common in females ${ }^{82}$ with an incidence of $8 \%$ compared to $0.6 \%$ in males. ${ }^{18}$ Diabetes is commonly associated with CTS and studies have found about $11 \%$ in T1DM, $12 \%$ in T2DM and $8 \%$ in controls. ${ }^{7}$ CTS is associated with age and the duration of diabetes. A meta-analysis suggested that both T1DM and T2DM are risk factors for $\mathrm{CTS}^{83} \mathrm{~A}$ study found that the prevalence increased in both T1DM and T2DM compared to controls (26.7 and $15.0 \%$ versus 3.3 and $5 \%, \mathrm{p}<0.01$ and non-significant). ${ }^{73}$

\section{Flexor tenosynovitis}

Flexor tenosynovitis is also known as stenosing tenosynovitis or trigger finger. ${ }^{84}$ It is caused due to fibrous proliferation in the tendon sheath, which leads to limited movement around the fingers and hands $^{84} \mathrm{In} \mathrm{PWD}$, the incidence of flexor tenosynovitis ranges from 10 to $20 \%{ }^{84}$ and is linked with age and the duration of diabetes. A study observed an increased incidence in those with impaired glucose tolerance. ${ }^{85}$ In both $\mathrm{T} 1 \mathrm{DM}$ and $\mathrm{T} 2 \mathrm{DM}$, the prevalence increased compared to controls ( 23.3 and $16.7 \%$ versus 0.0 and $3.3 \%$ ), $\mathrm{p}<0.01$ and $\mathrm{p}<0.05^{73}$ and in T2DM, the prevalence also increased with age $(\mathrm{p}<0.05){ }^{73}$

\section{Causes of musculoskeletal disorders and pain in diabetes}

The prevalence of musculoskeletal disorders of all types was $58.15 \%(95 \%$ CI $41.4,73.9)$, as reported in a systematic review. ${ }^{86} \mathrm{PWD}$ commonly have an increased risk of having musculoskeletal pain, ${ }^{22}$ which may be related to neuropathy, connective tissue disorders, ${ }^{64}$ osteoporosis, reduced insulin-like growth factor 1, vasculopathy, obesity, sedentary lifestyle or due to these factors combined. ${ }^{87} \mathrm{LJM}$ 
is associated with microvascular complications of diabetes in patients with T1DM..$^{81,88,89}$ In people with T2DM, musculoskeletal pain is especially common. ${ }^{87}$ A population-based retrospective cohort study demonstrated a significantly higher 10-year cumulative incidence of musculoskeletal pain in T2DM in both genders and in all age groups compared to controls $(\mathrm{p}<0.05) .{ }^{87}$ The relative risk $(\mathrm{RR})$ for all age groups and genders was $>1$ and was increased in females compared to males. ${ }^{87}$ Similarly, a previous study also found musculoskeletal pain in patients with T2DM, which was 1.7-2.1 times more than controls. ${ }^{20}$ Researchers have suggested that pain can also be due to osteoporosis, which has also been associated with T2DM. ${ }^{90}$ An earlier study had also reported that pain in the arms, hands, knees and/or hips and lowback pain had a significant relation with BMI $(\mathrm{p}<0.005) .{ }^{20}$ Impaired QoL, a sedentary lifestyle and reduced physical function $(\mathrm{p}<0.05)$ were related with low back pain, which was found less in males compared to females $(\mathrm{p}<0.001) .{ }^{20}$

\section{Hyperglycaemia}

Several studies have suggested that hyperglycaemia may play a vital role in musculoskeletal disorders and pain. ${ }^{24}$ Diabetes causes a disturbance in insulin metabolism that leads to hyperglycaemia, which commonly leads to other complications. ${ }^{91}$ Impaired insulin action on target tissues can be a result of faults in insulin secretion and/or decreased tissue responses to insulin, which may cause abnormalities in carbohydrate, fat and protein metabolism. ${ }^{2}$ Hyperglycemia may induce chronic inflammation that can lead to systemic changes in body organs. ${ }^{92}$ Studies have reported association of diabetes and hyperglycaemia with OA. ${ }^{93,94} \mathrm{OA}$ was found to have correlations with inadequate glycaemic control and longer duration of diabetes. ${ }^{11-13}$ Moderate hyperglycaemia has been linked with higher uric acid levels in individuals with gout. ${ }^{95}$ Chronic hyperglycaemia may also cause impaired growth and susceptibility to certain infections, such as feet and hand infections, in PWD. ${ }^{2}$

\section{Advanced glycation end-stage products}

Advanced glycation end products (AGEs) are harmful compounds and can be produced as a result of hyperglycaemia that can affect cartilage and bone health. ${ }^{65}$ A combination of protein or fat with glucose may lead to the formation of AGEs, which are linked with diabetic microvascular complications. ${ }^{96}$ AGEs can collect in the joints and surrounding areas, and cause increase the fragility of bones, cartilage stiffness and cause pain. ${ }^{97}$ They can alter the structure of proteins and may damage the intracellular and extracellular structural proteins, such as collagen. AGEs may also act through specific receptors. Studies have reported that they can alter cellular functions by attaching to RAGE (receptor for advanced glycation end products) and produce cellular signalling outcomes such as activation of some signalling mechanisms that may cause cell stress, cellular dysfunction and damage. ${ }^{65}$

\section{Inflammation}

Inflammation of a low-grade has been found that exists in diabetes $^{98}$ and also in OA.99 Inflammatory arthritis such as RA and psoriatric arthritis have both been linked with diabetes. ${ }^{100}$ In PWD, inflammatory markers are also found, that are also high in people who have inflammatory arthritis. ${ }^{100}$ Diabetes may trigger inflammation which causes progressive joint damage, stiffness and pain, such as in RA. A study reported that diabetes was associated with $55 \%$ elevated odds of having RA however, in this study RA was reported by $15.1 \%$ and $7.6 \%$ of the participants with and without diabetes. ${ }^{101}$
In RA, inflammation of joints and other tissues have been associated with nociceptive pain. ${ }^{102}$ Inflammation in RA can cause damage to the joints and more inflammation as in RA can increase IR and promote T2DM. Studies have suggested that inflammation may increase the risks of T2DM and OA and may also be a result of dyslipidaemia and visceral obesity. ${ }^{97,103}$

\section{Connective tissue}

Diabetes has major effects on connective tissues, which significantly impacts tendons, ligaments, bones and cartilage. ${ }^{104}$ An increased prevalence of connective tissue and musculoskeletal disorders has been observed in PWD. ${ }^{1}$ The alterations observed in connective tissue in PWD have suggested that various factors can lead to musculoskeletal pain. ${ }^{1}$ The cartilage extracellular matrix can alter due to metabolic abnormalities linked with diabetes. ${ }^{37}$ The periarticular and skeletal systems may also get altered due to diabetes. ${ }^{64}$ Abnormal deposition of collagen in the periarticular connective tissues can alter the extracellular matrix and cause damage. ${ }^{10,105}$ Some studies in animal models of diabetes have shown a decrease in collagen production ${ }^{58}$ and increased catabolism of proteoglycans, ${ }^{106}$ which supports these findings. Faults related to disturbed glucose metabolism, such as increased non-enzymatic glycosylation of collagen, ${ }^{107,108}$ crosslinking of collagen and resistance to enzymatic degradation, increased hydration of collagen ${ }^{109}$ and changed collagen synthesis, may contribute to some musculoskeletal disorders and pain in PWD ${ }^{65}$

\section{Inflammatory cytokines}

Certain pro-inflammatory cytokines such as tumor necrosis factor alpha [TNF- $\alpha$ ], interleukin-6 [IL-6] and interleukin-1 beta (IL-1 $\beta$ ) have been found to be involved in musculoskeletal pain. TNF- $\alpha$, a pleiotropic cytokine, is known to facilitate pathological conditions such as inflammation, arthritis, IR, immunomodulation, autoimmune diseases and apoptosis. ${ }^{18}$ Bone resorption and osteoclastogenesis are increased by TNF- $\alpha$ when the receptor activator of nuclear factor kappa B ligand (RANKL) is present. ${ }^{18}$ Inflammatory response is increased by IL- 6 , which activates the immune system. A number of studies have shown that some immunoregulatory components such as IL-6 and TNF- $\alpha$ are linked in musculoskeletal disorders such as RA, IR and T2DM. ${ }^{110,111}$ They play an important role through intracellular pathways of signal transduction for producing cytokines, some enzymes and other inflammatory compounds. ${ }^{12}$ The intracellular pathways get activated and causes inflammation and catabolism to increase in joint tissues, ${ }^{113,114}$ which causes a loss of metabolic homeostasis. ${ }^{112}$ A study reported higher synovitis scores $(\mathrm{p}=0.024)$ and a significant increase in the levels of IL-6 $(p=0.003)$ in the synovial fluid of knee joints of PWD, in comparison with patients without diabetes. ${ }^{115}$

\section{Obesity}

In recent years, diabetes and obesity have increased at an alarming rate globally. ${ }^{7}$ The risk of T2DM as well as forms of arthritis such as $\mathrm{OA}$, gout and RA may be increased due to obesity. ${ }^{32}$ Musculoskeletal pain had an association with raised BMI, decreased quality of life (QoL), lower physical function and being physically active, in a previous study on 940 patients with T2DM..$^{20}$ Associations between $\mathrm{OA}$ and T2DM have also been found which were linked with the underlying risk factors of obesity and age. ${ }^{99}$ In OA, damage to the joints may be due to excess pressure on the joints caused by obesity and joint injury. ${ }^{29} \mathrm{~A}$ higher incidence of RA found in a study was likely to be driven by obesity, comorbidities and lifestyle factors. ${ }^{116}$ 
Dyslipidemia, hyperuricaemia, obesity and increased levels of serum insulin were found to be increased in patients with DISH compared to controls. ${ }^{18}$ An increased production of TNF-alpha, IL-6, leptin and resistin has been found in obesity, which may contribute to IR. ${ }^{117}$ Conversely, there is a decrease in the plasma levels and expression of the insulin-sensitizing effector, adiponectin, in obesity. ${ }^{117}$

\section{Discussion}

This review looked at how diabetes affects musculoskeletal disorders, for a better understanding of the impact it can have on PWD. Musculoskeletal disorders are common in T1DM and T2DM and several studies have reported that PWD have an elevated risk of having musculoskeletal pain. ${ }^{118}$ Diabetes has also been associated with elevated odds of OA, RA and osteoporosis. ${ }^{22}$ A cross-sectional study reported that long duration of diabetes and dyslipidaemia were associated with raised prevalence of hand abnormalities $(\mathrm{p}=0.017$; $\mathrm{p}=0.019) .{ }^{8}$ Moreover, an increase was observed in the prevalence of soft tissue hand lesions in both T1DM and T2DM, compared to controls. ${ }^{73}$ Researchers have suggested that disorders such as AC may have other complications of diabetes such as LJM, than controls. Changes in connective tissue could be the underlying mechanism that causes disorders such as LJM, DC and AC. ${ }^{65}$ Insufficient control of diabetes can damage nerves, muscles and bones and cause musculoskeletal pain over time. ${ }^{119}$ In PWD, musculoskeletal pain can occur due to a number of reasons, such as vasculopathy, connective tissue disorders, neuropathy or a combination of them. Neuropathy may be the underlying cause of the increased incidence of musculoskeletal disorders with associated pain in PWD. ${ }^{65}$ The harmful effects of hyperglycaemia can cause diabetic neuropathy. ${ }^{120}$ Neuropathic joints are complications of diabetes and are commonly found mainly in the foot and ankle of PWD. ${ }^{121}$ A number of studies have found that RA has an association with neuropathic pain. ${ }^{122-124}$ The pain may also be a consequence of the complications of diabetic polyneuropathy. ${ }^{22}$ Neuropathic pain can increase due to central sensitization..$^{43}$ Although a study found a significant increase in the prevalence of musculoskeletal pain in patients with T2DM, only a weak correlation was found between the intensity of pain and peripheral inflammation. ${ }^{29}$

Studies have shown that hyperglycaemia can cause cell damage and induce inflammation by various mechanisms. A loss of BMD in PWD may be due to an increase in bone resorption and decrease in bone formation when diabetes is not controlled effectively. ${ }^{125}$ Chronic inflammation due to diabetes has been found to lead to RA. An increased risk for diabetes associated with RA was found in a population-based study ${ }^{126}$ and another study showed that RA increased the risk for diabetes by about $50 \% .{ }^{51}$ Pain is frequently considered a marker of inflammation and in patients with RA it may be caused due to joint inflammation or secondary OA. ${ }^{127}$ Low-grade inflammation has been found to be associated with diabetes ${ }^{98}$ and OA. ${ }^{99}$ However, other than inflammation, some studies emphasize that the association between diabetes and OA could be due to AGEs and oxidative stress. ${ }^{92,127}$ The accumulation of AGEs may cause musculoskeletal pain in PWD in joints and their surrounding tissues. ${ }^{117}$ Hyperglycaemia may also raise the levels of plasma insulin significantly. High levels of insulin or insulin-like growth factors (IGF-1, IGF-2) due to hyperglycaemia, can promote new bone growth or calcification of bones, especially in obese patients with T2DM, which may explain a normal/high BMD found in T2DM patients with hyperinsulinaemia ${ }^{65}$ High levels of insulin and IGF-1 can lead to calcification and ossification of ligaments and areas that are exposed to mechanical stress. ${ }^{128}$ Mechanical stress can cause oxidative damage, as indicated in previous studies. ${ }^{129}$ Some studies have found that hyperglycaemia can lead to joint inflammation and cartilage degradation not only through AGEs and oxidative stress, but also inflammatory mediators. ${ }^{127}$ A significant synovitis observed in OA may have been due to the increased levels of inflammatory cytokines, prostaglandins and adipokines found in diabetic tissues. ${ }^{37}$

There is evidence that there are links between inflammations, TNF- $\alpha$, IL- 6 and RANKL, which regulates osteoclastogenesis. ${ }^{19}$ $\mathrm{TNF} \alpha$ stimulates resorption, inhibits the synthesis of proteoglycan in cartilage and inhibits the synthesis of proteoglycans and type II collagen by chondrocytes. ${ }^{130,131}$ This may lead to the death of chondrocytes and prevent cartilage regeneration. Increased concentrations of IL-6 $(\mathrm{p}=0.003)$ found in the synovial fluid of PWD and knee OA was likely to have been the reason for a significant increase in the intensity of pain. ${ }^{15}$ In another study, increased hand pain in erosive OA along with worsening of pain was probably due to diabetes and/or synovitis. ${ }^{23}$ In contrast, a Finnish self-reported study did not find diabetes to be associated with pain intensity in older males or females. ${ }^{132}$ However, they did not measure cytokine levels, and this was a cross-sectional study, a cause- effect relationship cannot be assumed. Moreover, selfreported data may have certain inaccuracies and additionally, the study population was only from one area and therefore the results may not be generalizable to a larger population. Chronic hyperglycaemia may also cause susceptibility to certain infections in PWD, which may be caused due to arterial disease, diabetic neuropathy or obesity. ${ }^{1,2}$ Other than associations with inadequate glycaemic control, raised BMI, age and duration of diabetes, musculoskeletal conditions and pain in diabetes have been found to be associated with reductions in QoL, physical function and being physically active. ${ }^{20}$ Pain in the hands, arms, knees, hips and low-back pain have been associated with BMI $(\mathrm{p}<0.005) .{ }^{20}$ Studies have confirmed that in RA, diabetes occurred more often with increasing BMI and age. ${ }^{119}$

\section{Future implications}

Future studies are needed to clarify the links between body composition, TNF- $\alpha$, inflammation, musculoskeletal pain and IR. Studies have indicated that an optimal glycaemic control, control of inflammation and insulin sensitivity may decrease the risk of developing diabetes and musculoskeletal disorders associated with diabetes. Moreover, blockade of TNF- $\alpha$ may improve IR and lipid profiles and additionally, cytokines could be important targets for therapies. An improved understanding of the abnormalities of connective tissue metabolism in diabetes is needed. The underlying mechanisms that underlie diabetic neuropathic pain can lead to new or improved therapies to optimize pain control and may lead to significant improvements in clinical care of PWD.

\section{Conclusion}

In conclusion, musculoskeletal disorders and pain are common in PWD. Diabetes is associated with musculoskeletal disorders like $\mathrm{OA}, \mathrm{RA}$, and osteoporosis and fibromyalgia syndrome along with pain. Insufficient glycaemic control can lead to worsening of these disorders in PWD, which clearly needs attention in clinical practice. This review suggests having an increased focus on musculoskeletal disorders in PWD in clinical practice. Early management of musculoskeletal problems in PWD and maintaining good glycaemic control can prevent or improve musculoskeletal disorders, pain, morbidity, mortality and improve QoL. Future studies to understand how diabetes relates with connective tissue metabolism, cytokines 
and obesity for better patient care, prevention or management and treatment, are desirable.

\section{Acknowledgments}

None.

\section{Conflicts of interest}

The authors declare no conflict of interest.

\section{Funding}

None.

\section{References}

1. Nieves-Plaza M, Castro-Santana LE, Font YM, et al. Association of hand or knee osteoarthritis with diabetes mellitus in a population of hispanics from Puerto Rico. J Clin Rheumatol. 2013;19(1):1-6.

2. American Diabetes Association (ADA). Diagnosis and classification of diabetes mellitus. Diabetes care. 2013;36(Suppl 1):S67-S74.

3. World Health Organization. Diabetes. 2020.

4. Guariguata L, Whiting DR, Hambleton I, et al. Global estimates of diabetes prevalence for 2013 and projections for 2035. Diabetes Res Clin Pract. 2014;103(2):137-149.

5. Centers for Disease Control \& Prevention. National diabetes statistics report: estimates of diabetes and its burden in the United States, 2014. Atlanta, GA: US Department of Health and Human Services. 2014.

6. Diabetes in the UK 2010. Key statistics on diabetes.

7. Pandey R, Kumar N, Paroha S, et al. Impact of obesity and diabetes on arthritis: an update. Health (Irvine Calif). 2013;5(1):143-156.

8. Majjad A, Errahali Y, Toufik H, et al. Musculoskeletal disorders in patients with diabetes mellitus: a cross-sectional study. Int $J$ Rheumatol. 2018:3839872

9. Merashli M, Chowdhury TA, Jawad ASM. Musculoskeletal manifestations of diabetes mellitus. QJM: An International Journal of Medicine. 2015;108(11):853-857.

10. Mueller MJ. Musculoskeletal impairments are often unrecognized and underappreciated complications from diabetes. Phys Thert. 2016; 96(12):1861-1864

11. Swierkot J, Guszecka-Marczyńska K, Sowińsk D, et al. Rheumatic disorders in diabetes mellitus. Pol Merkur Lekarski. 2005;19(114):843847.

12. Del Rosso A, Cerinic MM, De Giorgio F, et al. Rheumatological manifestations in diabetes mellitus. Curr Diabetes Rev. 2006;2(4):455466.

13. Douloumpakas I, Pyrpasopoulou A, Triantafyllou A, et al. Prevalence of musculoskeletal disorders in patients with type 2 diabetes mellitus: a pilot study. Hippokratia. 2007;11(4):216-218.

14. Murphy L, Schwartz TA, Helmick CG, et al. Lifetime risk of symptomatic knee osteoarthritis. Arthritis Rheum. 2008;59(9):1207-1213.

15. The impact of musculoskeletal disorders on Americans-opportunities for action. Bone and Joint Initiative USA. 2016.

16. James SL, Abate D, Abate $\mathrm{KH}$, et al. Global, regional, and national incidence, prevalence, and years lived with disability for 354 diseases and injuries for 195 countries and territories, 1990-2017: a systematic analysis for the Global Burden of Disease Study 2017. Lancet. 2018;392(10159):1789-1858.
17. Crispin JC, Alcocer-Varela J. Rheumatic manifestations of diabetes mellitus. Am J Med. 2003;114(9):753-757.

18. Sözen T, Başaran NÇ, Tınazlı M, et al. Musculoskeletal problems in diabetes mellitus. Eur J Rheumatol. 2018;5(4):258-265.

19. Laranjo L, Neves AL, Costa A, et al. Facilitators, barriers and expectations in the self-management of type 2 diabetes--a qualitative study from Portugal. Eur J Gen Pract. 2015;21(2):103-110.

20. Molsted S, Tribler J, Snorgaard O. Musculoskeletal pain in patients with type 2 diabetes. Diabetes Res Clin Pract. 2012;96(2):135-140.

21. The National Institute for Health \& Care Excellence (NICE). The Joint pain advisor approach for knee and hip Pain. 2017.

22. Rehling T, Bjørkman AD, Andersen MB, et al. Diabetes is associated with musculoskeletal pain, osteoarthritis, osteoporosis, and rheumatoid arthritis. Journal of Diabetes Research. 2019:6324348.

23. King KB, Findley TW, Williams AE, et al. Veterans with diabetes receive arthroplasty more frequently and at a younger age. Clin Orthop Relat Res. 2013;471:3049-3054.

24. Schreiber AK, Nones CF, Reis RC, et al. Diabetic neuropathic pain: physiopathology and treatment. World J Diabetes. 2015;6(3):432-444.

25. Arkkila PE, Kantola IM, Viikari JS, et al. Shoulder capsulitis in type I and II diabetic patients: association with diabetic complications and related diseases. Ann Rheum Dis. 1996;55(12):907-914.

26. Diabetes.co.uk. 2019.

27. Diabetes.co.uk. 2019

28. Bayne O, Lu EJ. Diabetic charcot's arthropathy of the wrist. case report and literature review. Clin Orthop Relat Res. 1998;357:122-126.

29. Molines L, Darmon P, Raccah D. Charcot's foot: newest findings on its pathophysiology, diagnosis and treatment. Diabetes Metab. 2010;36(4):251-255

30. Hartemann-Heurtier A, Ha Van G, Grimaldi A. The charcot foot. Lancet 2002;360:1776-1779.

31. Collange C, Burde MA. Musculoskeletal problems of neurogenic origin. Baillieres Best Pract Res Clin Rheumatol. 2000;14(2):325-343.

32. Diabetes.co.uk. 2019

33. Wu Q, Zhu M, Rosier RN, et al. Beta-catenin, cartilage, and osteoarthritis. Ann N Y Acad Sci. 2010;1192(1):344-350.

34. Louati K, Vidal C, Berenbaum F, et al. Association between diabetes mellitus and osteoarthritis: systematic literature review and meta-analysis. RMD Open. 2015;1(1):e00077.

35. Williams MF, London DA, Husni EM, et al. Type 2 diabetes and osteoarthritis: a systematic review and meta-analysis. $J$ Diabetes Complications. 2016;30(5):944-950.

36. Eymard F, Parsons C, Edwards MH, et al. Diabetes is a risk factor for knee osteoarthritis progression. Osteoarthritis Cartilage. 2015;23(6):851-859.

37. King KB, Rosenthal AK. The adverse effects of diabetes on osteoarthritis: update on clinical evidence and molecular mechanisms. Osteoarthritis Cartilage. 2015;23(6):841-850.

38. Sturmer T, Brenner H, Brenner RE, et al. Non-insulin dependent diabetes mellitus (NIDDM) and patterns of osteoarthritis. the ulm osteoarthritis study. Scand J Rheumatol. 2001;30(3):169-171.

39. Veronese N, Cooper C, Reginster JY, et al. Type 2 diabetes mellitus and osteoarthritis. Semin Arthritis Rheum. 2019;49(1):9-19.

40. Firestein GS. Evolving concepts of rheumatoid arthritis. Nature. 2003;423(6937):356-361. 
41. McInnes IB, Schett G. The pathogenesis of rheumatoid arthritis. $N$ Engl J Med. 2011;365(23):2205-2219.

42. Diabetes.co.uk. 2019

43. Ahmed S, Magan T, Vargas M, et al. Use of the painDETECT tool in rheumatoid arthritis suggests neuropathic and sensitization components in pain reporting. J Pain Res. 2014;7:579-588.

44. Cross M, Smith E, Hoy D, et al. The global burden of rheumatoid arthritis: estimates from the global burden of disease 2010 study. Ann Rheum Dis. 2014;73(7):1316-1322.

45. Han C, Robinson DW, Hackett MV. Cardiovascular disease and risk factors in patients with rheumatoid arthritis, psoriatic arthritis, and ankylosing spondylitis. $J$ Rheumatol. 2006;33(11):2167-2172.

46. Rincon ID, Williams K, Stern MP, et al. High incidence of cardiovascula events in a rheumatoid arthritis cohort not explained by traditional cardiac risk factors. Arthritis Rheumatol. 2001;44(12):2737-2745.

47. Bland JH, Frymoyer JW, Newberg AH, et al. Rheumatic syndromes in endocrine disease. Semin Arthritis Rheum 1979;9(1):23-65.

48. Sturfelt G, Leden E, Nived O. Hand symptoms associated with diabetes mellitus. An investigation of 765 patients based on a questionnaire. Acta Med Scand. 1981;210(1-2):35-38.

49. Solomon DH, Love TJ, Canning C, et al. Risk of diabetes among patients with rheumatoid arthritis, psoriatic arthritis and psoriasis. Ann Rheumatol Dis. 2010;69(12):2114-2117.

50. Kuo CF, Grainge MJ, Valdes AM, et al. Familial aggregation of rheumatoid arthritis and co-aggregation of autoimmune diseases in affected families: a nationwide population-based study. Rheumatology (Oxford). 2017;56(6):928-933.

51. Kim SC, Liu J, Solomon DH. Risk of incident diabetes in patients with gout: a cohort study. Arthritis Rheumatol. 2015;67(1):273-280.

52. Rho YH, Lu N, Peloquin CE, et al. Independent impact of gout on the risk of diabetes mellitus among women and men: a population-based, BMImatched cohort study. Ann Rheum Dis. 2016;75(1):91-95.

53. Rodríguez G, Soriano LC, Choi HK. Impact of diabetes against the future risk of developing gout. Ann Rheum Dis. 2010;69(12):2090-2094.

54. Chen JH, Yeh WT, Chuang SY, et al. Gender-specific risk factors for incident gout: a prospective cohort study. Clin Rheumatol. 2012;31(2):239-245.

55. Diabetes.co.uk. 2019

56. Suppiah R, Dissanayake A, Dalbeth N. High prevalence of gout in patients with type 2 diabetes: male sex, renal impairment, and diuretic use are major risk factors. $N Z$ Med J. 2008;121(1283):43-50.

57. Kodama S, Saito K, Yachi Y, et al. Association between serum uric acid and development of type 2 diabetes. Diabetes Care. 2009;32(9):17371742 .

58. Umpierrez GE, Zlatev T, Spanheimer RG. Correction of altered collagen metabolism in diabetic animals with insulin therapy. Matrix. 1989;9(4):336-342

59. Trujillo-Santos AJ. Diabetic muscle infarction: an underdiagnosed complication of long-standing diabetes. Diabetes Care. 2003;26(1):211215

60. Morcuende JA. Dobbs MB, Crawford H, et al. Diabetic muscle infarction Iowa Orthop J. 2000;20:65-74.

61. Smith LL. Burnet SP, McNeil JD. Musculoskeletal manifestations of diabetes mellitus. Br J Sports Med. 2003;37(1):30-35.
62. Zaidi SA, Chhetri SK, Lekwuwa G, et al. An unusual presentation of diabetic amyotrophy: myoclonus. BMJ Case Rep. 2013:bcr2012008245.

63. Grodman I, Buskila D, Arnson Y, et al. Understanding fibromyalgia and its resultant disability. IMAJ. 2011;13(12):769-772.

64. Lichtenstein A, Tiosano S, Comaneshter D, et al. Cross-sectional analysis of the associations between fibromyalgia and diabetes mellitus. Reumatologia. 2018;56(5):275-278.

65. Arkkila PE. Gautier JF. Musculoskeletal disorders in diabetes mellitus: an update. Best Pract Res Clin Rheumatol. 2003;17(6):945-970.

66. Vezyroglou G, Mitropoulos A, Antoniadis CA. Metabolic syndrome in diffuse idiopathic skeletal hyperostosis. A controlled study. J Rheumatol. 1996;23(4):672-676.

67. Grazina R, Teixeira S, Ramos R, et al. Dupuytren's disease: where do we stand? EFORT Open Rev. 2019;4(2):63-69.

68. Geoghegan JM, Forbes J, Clark DI, et al. Dupuytren's disease risk factors. J Hand Surg Br. 2004;29(5):423-426.

69. Fossati P, Romon M, Vennin P. Dupuytren's contracture and diabetes mellitus. Ann Chir Main. 1982;1(4):351-354.

70. Stradner F, Ulreich A, Pfeiffer KP. Dupuytren's contracture as a concomitant disease in diabetes mellitus. Wien Med Wochenschr. 1987;137(4):89-92.

71. Broekstra DC, Groen H, Molenkamp S, et al. A systematic review and meta-analysis on the strength and consistency of the associations between dupuytren disease and diabetes mellitus, liver disease, and epilepsy. Plast Reconstr Surg. 2018;141(3):367e-379e.

72. Jennings AM, Milner PC, Ward JD. Hand abnormalities are associated with the complications of diabetes in type 2 diabetes. Diabet Med. 1989;6(1):43-47.

73. Renard E, Jacques D, Chammas M, et al. Increased prevalence of soft tissue hand lesions in type 1 and type 2 diabetes mellitus: various entities and associated significance. Diabete Metab. 1994;20(6):513-521.

74. Balci N, Balci MK, Tuzuner S. Shoulder adhesive capsulitis and shoulder range of motion in type II diabetes mellitus: association with diabetic complications. J Diabetes Complications. 1999;13(3):135-140.

75. Mavrikakis ME, Drimis S, Kontoyannis DA, et al. Calcific shoulder periarthritis (tendinitis) in adult onset diabetes mellitus: a controlled study. Ann Rheum Dis. 1989;48(3):211-214.

76. Valderrábano RJ, Linares MI. Diabetes mellitus and bone health: epidemiology, etiology and implications for fracture risk stratification. Clin Diabetes Endocrinol. 2018;4:9.

77. Janghorbani M, Feskanich D, Willett WC, Hu F. Prospective study of diabetes and risk of hip fracture: the nurses' health study. Diabetes Care. 2006;29(7):1573-1578.

78. Gerrits EG, Landman GW, Nijenhuis-Rosien L, et al. Limited joint mobility syndrome in diabetes mellitus: a mini review. World J Diabetes. 2015;6(9):1108-1112.

79. Crisp AJ, Heathcote JG. Connective tissue abnormalities in diabetes mellitus. J R Coll Physicians Lond. 1984;18(2):132-141.

80. Traisman HS, Traisman ES, Marr TJ, et al. Joint contractures in patients with juvenile diabetes and their siblings. Diabetes Care. 1978;1(6):360 361.

81. Rosenbloom AL, Silverstein JH, Lezotte DC, et al. Limited joint mobility in childhood diabetes indicates increased risk for microvascular disease. N Engl J Med. 1981;305(4):191-194. 
82. Gül Yurdakul F, Bodur H, Öztop Çakmak Ö, et al. On the severity of carpal tunnel syndrome: diabetes or metabolic syndrome. J Clin Neurol. 2015;11(3):234-240.

83. Pourmemari MH, Shiri R. Diabetes as a risk factor for carpal tunnel syndrome: a systematic review and meta-analysis. Diabet Med. 2016;33(1):10-16.

84. Ray G, Tall MA. Tenosynovitis. [Updated 2019 Jun 17]. In: StatPearls [Internet]. Treasure Island (FL): StatPearls Publishing; 2020 Jan.

85. Leden I, Svensson B, Sturfelt G, et al. Rheumatic hand symptoms as a clue to undiagnosed diabetes mellitus. Scand J Rheumatol. 1980;9(2):127128

86. Kaka B, Maharaj SS, Fatoye F. Prevalence of musculoskeletal disorder in patients with diabetes mellitus: a systematic review and meta-analysis. J Back Musculoskelet Rehabil. 2019;32(2):223-235.

87. Pai LW, Hung CT, Li SF, et al. Musculoskeletal pain in people with and without type 2 diabetes in Taiwan: a population-based, retrospective cohort study. BMC Musculoskeletal Disorders. 2015;16(1):364.

88. Frost D, Beischer W. Limited joint mobility in type 1 diabetic patients Associations with microangiopathy and subclinical macroangiopathy are different in men and women. Diabetes Care. 2001;24(1):95-99.

89. Arkkila PE, Kantola IM, Viikari JS. Limited joint mobility in type 1 diabetic patients: correlation to other diabetic complications. $J$ Intern Med. 1994;236(2):215-223.

90. Wimalawansa SJ. Vitamin D deficiency: effects on oxidative stress, epigenetics, gene regulation, and aging. Biology. 2019;8(2):30.

91. Alenazi AM, Alshehri MM, Alothman S, et al. The Association of diabetes with knee pain severity and distribution in people with knee osteoarthritis using data from the osteoarthritis initiative. Scientific report. 2020;10(1):3985.

92. Atayde SA, Yoshinari NH, Nascimento DP, et al. Experimental diabetes modulates collagen remodelling of joints in rats. Histol Histopathol. 2012;27(11):1471-1479.

93. Karvonen-Gutierrez CA, Sowers MR, Heeringa SG. Sex dimorphism in the association of cardiometabolic characteristics and osteophytesdefined radiographic knee osteoarthritis among obese and non-obese adults: NHANES III. Osteoarthritis Cartilage. 2012;20(7):614-621.

94. Schett G, Kiechl S, Bonora E et al. Vascular cell adhesion molecule 1 as a predictor of severe osteoarthritis of the hip and knee joints. Arthritis Rheum. 2009;60(8):2381-2389.

95. Choi HK, Ford ES. Prevalence of the metabolic syndrome in individuals with hyperuricemia. Am J Med. 2007;120(5):442-447.

96. Brownlee M. Biochemistry and molecular cell biology of diabetic complication. Nature. 2001;414(6865):813-882.

97. Courties A, Gualillo O, Berenbaum F, et al. Metabolic stress-induced joint inflammation and osteoarthritis. Osteoarthritis Cartilage. 2015;23(11):1955-1965.

98. Wang X, Bao W, Liu J, et al. Inflammatory markers and risk of type 2 diabetes: a systematic review and meta- analysis. Diabetes Care. 2013;36(1):166-175

99. Piva SR, Susko AM, Khoja SS, et al. Links between osteoarthritis and diabetes: implications for management from a physical activity perspective. Clin Geriatr Med. 2015;31(1):67-87,viii.

100. Dubreuil M, Rho YH, Man A, et al. Diabetes incidence in psoriatic arthritis, psoriasis and rheumatoid arthritis: a UK population-based cohort study. Rheumatology (Oxford). 2014;53(2):346-352.
01. Abhishek A, Doherty M, Kuo CF, et al. Rheumatoid arthritis is getting less frequent-results of a nationwide population-based cohort study. Rheumatology (Oxford). 2017;56(5):736-744.

102. Schaible HG. Nociceptive neurons detect cytokines in arthritis. Arthritis Res Ther. 2014;16(5):470

03. Collins KH, Herzog W, MacDonald GZ, et al. Obesity, metabolic syndrome, and musculoskeletal disease: common inflammatory pathways suggest a central role for loss of muscle integrity. Front Physiol. 2018;9:112.

104. Burner TW, Rosenthal AK. Diabetes and rheumatic diseases. Curr Opin Rheumatol. 2009;21(1):50-54.

105. Alikhani M, Alikhani Z, Boyd C, et al. Advanced glycation end products stimulate osteoblast apoptosis via the MAP kinase and cytosolic apoptotic pathways. Bone. 2007;40(2):345-353

106. Silberberg R, Hirshberg GE, Lesker P. Enzyme studies in the articular cartilage of diabetic rats and of rats bearing transplanted pancreatic islets. Diabetes. 1977;26(8):732-735.

107. Vishwanath V, Frank KE, Elments CA, et al. Glycation of skin collagen in type I diabetes mellitus: correlation with long-term complications. Diabetes. 1986;35(8):916-921.

108. Salmela PI, Oikarainen A, Pirttiaho H, et al. Increased non-enzymatic glycosylation and reduced solubility of skin collagen in insulin-dependent diabetic patients. Diabetes Res. 1989;11(3):115-120.

109. Kapoor A, Sibbitt WL Jr. Contractures in diabetes mellitus: the syndrome of limited joint mobility. Semin Arthritis Rheum. 1980;18(3):168-180.

110. Popa C, Netea MG, van Riel PL, et al. The role of TNF-alpha in chronic inflammatory conditions, intermediary metabolism, and cardiovascular risk. J Lipid Res. 2007;48(4):751-762.

111. Aggarwal BB. Tumour necrosis factors receptor associated signalling molecules and their role in activation of apoptosis, JNK and NF-kappaB. Ann Rheum Dis. 2000;59(Suppl 1):i6-i16.

112. Wojdasiewicz P, Poniatowski ŁA, Szukiewicz D. The role of inflammatory and anti-inflammatory cytokines in the pathogenesis of osteoarthritis. Mediators Inflamm. 2014;561459.

113. Roman-Blas JA, Jimenez SA. NF- $\kappa$ B as a potential therapeutic target in osteoarthritis and rheumatoid arthritis. Osteoarthritis and Cartilage. 2006;14(9):839-848.

114. Marcu KB, Otero M, Olivotto E, et al. NF-KaapaB signaling: multiple angles to target OA. Current Drug Targets. 2010;11(5):599-613.

115. Eitner A, Pester J, Vogel F, et al. Pain sensation in human osteoarthritic knee joints is strongly enhanced by diabetes mellitus. Pain. 2017;158(9):17431753.

116. Stavropoulos-Kalinoglou A, Metsios GS, Koutedakis Y, et al. Obesity in rheumatoid arthritis, Rheumatology (Oxford). 2011;50(3):450-462.

117. Bastard JP, Maachi M, Lagathu C, et al. Recent advances in the relationship between obesity, inflammation, and insulin resistance. Eur Cytokine Netw. 2006;17(1):4-12.

118. Herbert MS, Varley AL, Andreae SJ, et al. Association of pain with HbA1c in a predominantly black population of community-dwelling adults with diabetes: a cross-sectional analysis. Diabet Med. 2013;30(12):1466-1471.

119. Albrecht K, Ramos AL, Hoffmann F, et al. High prevalence of diabetes in patients with rheumatoid arthritis: results from a questionnaire survey linked to claims data. Rheumatology (Oxford). 2018;57(2):329-336.

120. Lebiedz-Odrobina D, Kay J. Rheumatic manifestations of diabetes mellitus. Rheum Dis Clin North Am. 2010;36(4):681-699. 
121. Wyatt LH, Ferrance RJ. The musculoskeletal effects of diabetes mellitus. $J$ Can Chiropr Assoc. 2006;50(1):43-50.

122. Mendes SMD, Queiroz BLL, Santana LV, et al. Impact of different levels of disease activity in painful profile and quality of life in people with rheumatoid. Braz J Med Human Health. 2014;2(1):17-25.

123. Walsh DA, McWilliams DF. Mechanisms, impact and management of pain in rheumatoid arthritis. Nat Rev Rheumatol. 2014;10(10):581-592.

124. Koop SM, ten Klooster PM, Vonkeman HE, et al. Neuropathic-like pain features and cross-sectional associations in rheumatoid arthritis. Arthritis Res Ther. 2015;17(1):237.

125. Carson J, Clarke C. Dupuytren's contracture in pensioners at the royal hospital Chelsea. J R Coll Physicians Lond. 1993;27(1):25-27.

126. Chung CP, Oeser A, Solus JF. Prevalence of the metabolic syndrome is increased in rheumatoid arthritis and is associated with coronary atherosclerosis. Atherosclerosis. 2008;196(2):756-763.

127. Berenbaum F. Diabetes-induced osteoarthritis: from a new paradigm to a new phenotype. Ann Rheum Dis. 2011;70(8):1354-1356.
128. Denko CW, Boja B, Moskowitz RW. Growth promoting peptides in osteoarthritis and diffuse idiopathic skeletal hyperostosis--insulin, insulinlike growth factor-I, growth hormone. J Rheumatol. 1994;21(9):17251730 .

129. Liang X, Wang Z, Gao M, et al. Cyclic stretch induced oxidative stress by mitochondrial and NADPH oxidase in retinal pigment epithelial cells. BMC Ophthalmol. 2019;19:79.

130. Saklatvala J. Tumour necrosis factor $\alpha$ stimulates resorption and inhibits synthesis of proteoglycan in cartilage. Nature. 1986;322(6079)547-549.

131. Séguin CA, Bernier, SM. TNFalpha suppresses link protein and type II collagen expression in chondrocytes: role of MEK1/2 and NF-kappaB signaling pathways. J Cell Physiol. 2003;197(3):356-369.

132. Karjalainen M, Saltevo J, Tiihonen M, et al. Frequent pain in older people with and without diabetes-finnish community based study. BMC Geriatr. 2018;18(1):73. 